\title{
Applying Cubic B-Spline Quasi-Interpolation to Solve 1D Wave Equations in Polar Coordinates
}

\author{
Hossein Aminikhah and Javad Alavi \\ Department of Applied Mathematics, School of Mathematical Sciences, University of Guilan, P.O. Box 1914, Rasht 41938, Iran \\ Correspondence should be addressed to Hossein Aminikhah; hossein.aminikhah@gmail.com
}

Received 27 August 2013; Accepted 14 October 2013

Academic Editors: Y. Peng and J. G. Zhou

Copyright (C) 2013 H. Aminikhah and J. Alavi. This is an open access article distributed under the Creative Commons Attribution License, which permits unrestricted use, distribution, and reproduction in any medium, provided the original work is properly cited.

\begin{abstract}
We provide numerical solution to the one-dimensional wave equations in polar coordinates, based on the cubic B-spline quasiinterpolation. The numerical scheme is obtained by using the derivative of the quasi-interpolation to approximate the spatial derivative of the dependent variable and a forward difference to approximate the time derivative of the dependent variable. The accuracy of the proposed method is demonstrated by three test problems. The results of numerical experiments are compared with analytical solutions by calculating errors $L_{2}$-norm and $L_{\infty}$-norm. The numerical results are found to be in good agreement with the exact solutions. The advantage of the resulting scheme is that the algorithm is very simple so it is very easy to implement.
\end{abstract}

\section{Introduction}

The term "spline" in the spline function arises from the prefabricated wood or plastic curve board, which is called spline, and is used by the draftsman to plot smooth curves through connecting the known point. The use of spline function and its approximation play an important role in the formation of stable numerical methods. As the piecewise polynomial, spline, especially B-spline, have become a fundamental tool for numerical methods to get the solution of the differential equations. In the past, several numerical schemes for the solution of boundary value problems and partial differential equations based on the spline function have been developed by many researchers. As early in 1968 Bickley [1] has discussed the second-order accurate spline method for the solution of linear two-point boundary value problems. Raggett and Wilson [2] have used a cubic spline technique of lower order accuracy to solve the wave equation. Chawla et al. [3] solved the one-dimensional transient nonlinear heat conduction problems using the cubic spline collocation method in 1975. Rubin and Khosla [4] first proposed the spline alternating direction implicit method to solve the partial differential equation using the cubic spline and enhanced accuracy of the approximate solution of the second derivative to the same as that of the first derivative. Jain and Aziz [5] have derived fourth-order cubic spline method for solving the nonlinear two-point boundary value problems with significant first derivative terms. In recent years, El-Hawary and Mahmoud [6], Mohanty [7], Mohebbi and Dehghan [8], Zhu and Wang [9], Ma et al. [10], Dosti and Nazemi [11], Wang et al. [12], and other researchers [13-16] have derived various numerical methods for solution of partial differential equations based on the spline function.

The hyperbolic partial differential equations model the vibrations of structures (e.g., buildings, beams, and machines) and are the basis for fundamental equations of atomic physics.

The one-dimensional linear singular hyperbolic equation is given by

$$
U_{t t}=U_{r r}+\frac{\alpha}{r} U_{r}+g(r, t), \quad 0<r<1, t>0, \alpha=1,2,
$$

subject to the initial conditions

$$
u(r, 0)=\varphi_{0}(r), \quad u_{t}(r, 0)=\varphi_{1}(r), \quad 0<r<1,
$$

and Dirichlet boundary conditions at $r=0$ and $r=1$ of the form

$$
u(0, t)=f_{0}(t), \quad u(1, t)=f_{1}(t), \quad t \geq 0,
$$


where $u=u(r, t), t$ is time variable and $r$ is distance variable, and subscripts $r$ and $t$ denote differentiation. For $\alpha=1$ and $\alpha=2$, the equation above represents one-dimensional wave equation in cylindrical and spherical polar coordinates, respectively. We assume that $\varphi_{0}(r), \varphi_{1}(r)$, and $f_{0}(t), f_{1}(t)$ and their derivatives are continuous functions of $r$ and $t$, respectively.

Mohanty et al. [17] have a numerical solution equation (1). In this paper, we provide a numerical scheme to solve singular hyperbolic equation (1) using the derivative of the cubic B-spline quasi-interpolation to approximate the spatial derivative of the differential equations and utilize a forward difference to approximate the time derivative such as $[9,11]$ shown.

This paper is organized as follows. In Section 2, the univariate spline quasi-interpolants were introduced and we obtain the numerical schemes using cubic B-spline interpolation to solve singular hyperbolic equation (1). The stability of this method is studied in Section 3. Numerical experiments for various test problems are solved to assess the accuracy of the technique and the maximum absolute errors will be presented in Section 4. Finally, we give some concluded remarks in Section 5.

\section{Univariate Spline Quasi-Interpolants Applied to Singular Hyperbolic Equation}

According to recurrence relation of B-spline [18] the $j$ th Bspline of degree $d$ for the knot sequence $x:=\left(x_{j}\right)$ is denoted by $B_{j, d}$ or $B_{j}$ and is obtain by the rule

$$
B_{j, d}(r)=\omega_{j, d} B_{j, d-1}(r)+\left(1-\omega_{j+1, d}\right) B_{j+1, d-1}(r)
$$

with

$$
\omega_{j, d}(r)=\frac{r-x_{j}}{x_{j+d-1}-x_{j}}, \quad B_{j, 0}(r)= \begin{cases}1, & x_{j} \leq r<x_{j+1} \\ 0, & \text { othetwise. }\end{cases}
$$

Now assume that $R_{n}=\left\{r_{j}, j=0,1, \ldots, n\right\}$ is a uniform partition of interval $I=[a, b]$, where $r_{0}=a, r_{n}=b$ and with meshlength $h=(b-a) / n$ and consider that $X_{n}=\left(x_{j}, j=-d\right.$, $-d+1, \ldots, n+d)$ subject to $x_{-d}=x_{-d+1}=\cdots=x_{-1}=a$ and $x_{j}=r_{j}, 0 \leq j \leq n$, and $x_{n}=x_{n+1}=\cdots=x_{n+d}=$ $b$. Moreover suppose that $S=\left\{B_{j}, j=1,2, \ldots, n+d\right\}$ and that $B_{j}$ is the $\mathrm{B}$-spline of degree $d$ for the knot sequence $X_{n}$. We denote by $S_{d}\left(R_{n}\right)$ the space of splines of degree $d$ and $C^{d-1}$ on the uniform partition $R_{n}$. Let the B-spline basis of $S_{d}\left(R_{n}\right)$ be $S$. With these notations, the support of $B_{j}$ is $\operatorname{supp}\left(B_{j}\right)=\left[x_{j-d-1}, x_{j}\right]$. Figure 1 shows the thirteen B-splines for the knot sequence $X_{10}=(1,1,1,1,2,3,4,5,6,7,8,9$, $10,11,11,11,11)$. Note that in Figure $1 B(j)=B_{j}(r), d=3$.

In [19] univariate spline quasi-interpolants (abbreviation QIs) can be defined as operators of the form $Q_{d} f=$ $\sum_{j=1}^{n+d} \mu_{j}(f) B_{j}$. We denote by $\Pi_{d}$ the space of polynomials of total degree at most $d$. In general we impose that $Q_{d} p=p$ for all $p \in \Pi_{d}$. As a consequence of this property, the approximation order is $O\left(h^{d+1}\right)$ on smooth functions. According to [19],

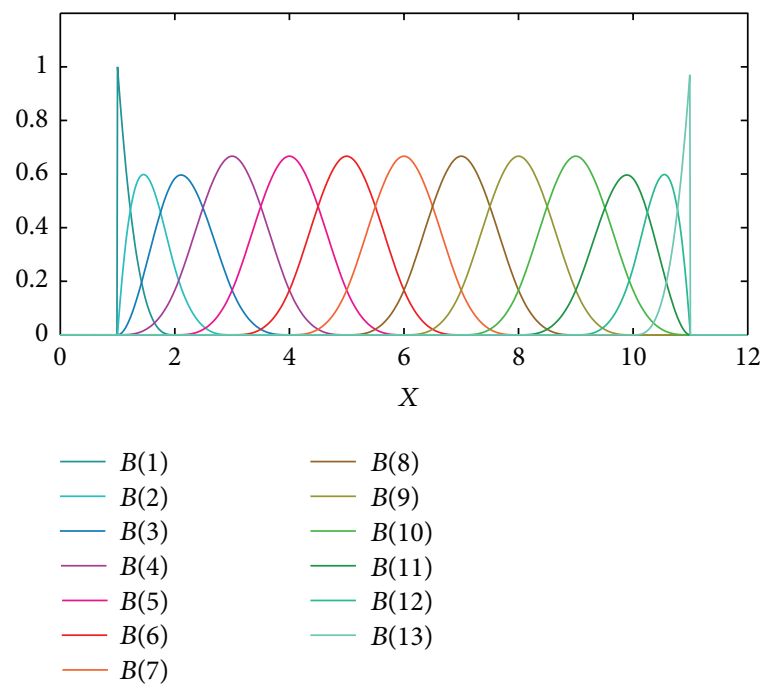

Figure 1: The B-splines for the knot sequence $X_{10}=$ $(1,1,1,1,2,3,4,5,6,7,8,9,10,11,11,11,11), B_{j, 3}, j=1,2, \ldots, 13$.

we assume that the coefficient $\mu_{j}(f)$ is a linear combination of discrete values of $f$ at some points in the neighborhood of $\operatorname{supp}\left(B_{j}\right)$.

The main advantage of QIs is that they have a direct construction without solving any system of linear equations. Moreover, they are local, in the sense that the value of $Q_{d} f$ depends only on values of $f$ in a neighborhood of $r$. Finally, they have a rather small infinity norm, so they are nearly optimal approximants [19]. For any subinterval $I_{k}=\left[r_{k-1}, r_{k}\right]$, $1 \leq k \leq n$, and for any function $f$,

$$
\left\|f-Q_{d} f\right\|_{\infty, I_{k}} \leq\left(1+\left\|Q_{d}\right\|_{\infty}\right) d_{\infty, I_{k}}\left(f, \Pi_{d}\right)
$$

where the distance of $f$ to polynomials is defined by

$$
d_{\infty, I_{k}}\left(f, \Pi_{d}\right)=\inf \left\{\|f-p\|_{\infty, I_{k}}, p \in \Pi_{d}\right\} .
$$

Here $\|f-p\|_{\infty, I_{k}}=\max _{r \in I_{k}}|f(r)-p(r)|$. Therefore, for $f \in$ $C^{d+1}(I)$, this implies that [19]

$$
\left\|f-Q_{d}\right\|_{\infty}=O\left(h^{d+1}\right)
$$

Since the cubic spline has become the most commonly used spline, we use cubic B-spline quasi-interpolation in this paper.

For cubic QI,

$$
Q_{3} f=\sum_{j=1}^{n+3} \mu_{j}(f) B_{j}
$$

and (4) implies that

$$
\left\|f-Q_{3}\right\|_{\infty}=O\left(h^{4}\right) .
$$


Let $f\left(R_{n}\right)=\left\{f_{j}=f\left(r_{j}\right), j=0,1, \ldots, n\right\}$; the coefficient functional are, respectively,

$$
\begin{gathered}
\mu_{1}(f)=f_{0}, \quad \mu_{2}(f)=\frac{1}{18}\left(7 f_{0}+18 f_{1}-9 f_{2}+2 f_{3}\right), \\
\mu_{j}(f)=\frac{1}{6}\left(-f_{j-3}+8 f_{j-2}-f_{j-1}\right), \quad 3 \leq j \leq n+1, \\
\mu_{n+2}(f)=\frac{1}{18}\left(2 f_{n-3}-9 f_{n-2}+18 f_{n-1}+7 f_{n}\right), \\
\mu_{n+3}(f)=f_{n} .
\end{gathered}
$$

For approximate derivatives of $f$ by derivatives of $Q_{3} f$ up to the order $h^{3}$, we can evaluate the value of $f$ at $x_{i}$ by $\left(Q_{3} f\right)^{\prime}=\sum_{j=1}^{n+3} \mu_{j}(f) B_{j}^{\prime}$ and $\left(Q_{3} f\right)^{\prime \prime}=\sum_{j=1}^{n+3} \mu_{j}(f) B_{j}^{\prime \prime}$. We set $Y=\left(f_{0}, f_{1}, \ldots, f_{n}\right)^{T}, Y^{\prime}=\left(f_{0}^{\prime}, f_{1}^{\prime}, \ldots, f_{n}^{\prime}\right)^{T}$, and
$Y^{\prime \prime}=\left(f_{0}^{\prime \prime}, f_{1}^{\prime \prime}, \ldots, f_{n}^{\prime \prime}\right)^{T}$ where $f_{j}^{\prime}=\left(Q_{3} f\right)^{\prime}\left(r_{j}\right), f_{j}^{\prime \prime}=$ $\left(Q_{3} f\right)^{\prime \prime}\left(r_{j}\right), j=1,2, \ldots, n$. Using (4), we can compute $B_{j}^{\prime}(r)$, $B_{j}^{\prime \prime}(r)$, and $d=3, j=1,2, \ldots, n+3$; see [11]. By solution of the linear systems

$$
\begin{aligned}
f_{i}^{\prime} & =\sum_{j=1}^{n+3} \mu_{j}(f) B_{j}^{\prime}\left(r_{i}\right), \quad i=0,1, \ldots, n, \\
f_{i}^{\prime \prime} & =\sum_{j=1}^{n+3} \mu_{j}(f) B_{j}^{\prime \prime}\left(r_{i}\right), \quad i=0,1, \ldots, n,
\end{aligned}
$$

we obtain the differential formulas for cubic B-spline QI as

$$
Y^{\prime}=\frac{1}{h} D_{1} Y, \quad Y^{\prime \prime}=\frac{1}{h^{2}} D_{2} Y,
$$

where $D_{1}, D_{2} \in \mathbb{R}^{(n+1) \times(n+1)}$ and obtain as follows:

$$
\begin{aligned}
& D_{1}=\left(\begin{array}{ccccccccc}
-\frac{11}{6} & 3 & -\frac{3}{2} & \frac{1}{3} & 0 & 0 & \ldots & 0 & 0 \\
-\frac{1}{3} & -\frac{1}{2} & 1 & -\frac{1}{6} & 0 & 0 & \ldots & 0 & 0 \\
\frac{1}{12} & -\frac{2}{3} & 0 & \frac{2}{3} & -\frac{1}{12} & 0 & \ldots & 0 & 0 \\
0 & \frac{1}{12} & -\frac{2}{3} & 0 & \frac{2}{3} & -\frac{1}{12} & \ldots & 0 & 0 \\
\vdots & \vdots & \vdots & \vdots & \vdots & \vdots & \vdots & \vdots & \vdots \\
0 & 0 & \ldots & \frac{1}{12} & -\frac{2}{3} & 0 & \frac{2}{3} & -\frac{1}{12} & 0 \\
0 & 0 & \ldots & 0 & \frac{1}{12} & -\frac{2}{3} & 0 & \frac{2}{3} & -\frac{1}{12} \\
0 & 0 & \ldots & 0 & 0 & \frac{1}{6} & -1 & \frac{1}{2} & \frac{1}{3} \\
0 & 0 & \ldots & 0 & 0 & -\frac{1}{3} & \frac{3}{2} & -3 & \frac{11}{6}
\end{array}\right), \\
& D_{2}=\left(\begin{array}{ccccccccc}
2 & -5 & 4 & -1 & 0 & 0 & \ldots & 0 & 0 \\
1 & -2 & 1 & 0 & 0 & 0 & \ldots & 0 & 0 \\
-\frac{1}{6} & \frac{5}{3} & -3 & \frac{5}{3} & -\frac{1}{6} & 0 & \ldots & 0 & 0 \\
0 & -\frac{1}{6} & \frac{5}{3} & -3 & \frac{5}{3} & -\frac{1}{6} & \ldots & 0 & 0 \\
\vdots & \vdots & \vdots & \vdots & \vdots & \vdots & \vdots & \vdots & \vdots \\
0 & 0 & \ldots & -\frac{1}{6} & \frac{5}{3} & -3 & \frac{5}{3} & -\frac{1}{6} & 0 \\
0 & 0 & \ldots & 0 & -\frac{1}{6} & \frac{5}{3} & -3 & \frac{5}{3} & -\frac{1}{6} \\
0 & 0 & \ldots & 0 & 0 & 0 & 1 & -2 & 1 \\
0 & 0 & \ldots & 0 & 0 & -1 & 4 & -5 & 2
\end{array}\right) .
\end{aligned}
$$


Now, we present the numerical scheme for solving onedimensional linear singular hyperbolic equation (1) with initial conditions (2) and Dirichlet boundary conditions (3) based on the cubic B-spline quasi-interpolant.

Discretizing (1),

$$
U_{t t}=U_{r r}+\frac{\alpha}{r} U_{r}+g(r, t),
$$

in time, we get

$$
u_{i}^{k+1}=\tau^{2}\left(\left(u_{r r}\right)_{i}^{k}+\frac{\alpha}{r_{i}}\left(u_{r}\right)_{i}^{k}+g\left(r_{i}, t_{k}\right)\right)+2 u_{i}^{k}-u_{i}^{k-1},
$$

where $u_{i}^{k}$ is the approximation of the value $u(r, t)$ at $\left(r_{i}, t_{k}\right)$, $t_{k}=k \tau$, and $\tau$ is the time step. Then, we use the derivatives of the cubic B-spline quasi-interpolant $Q_{3} u\left(r_{i}, t_{k}\right)$ to approximate $\left(u_{r}\right)_{i}^{k}$ and $\left(u_{r r}\right)_{i}^{k}$.

Assume that $U^{k}=\left(u_{0}^{k}, u_{1}^{k}, \ldots, u_{n}^{k}\right)$ is known for the nonnegative integer $k$. We set unknown vectors as

$$
\begin{aligned}
U_{r}^{k} & =\left(\left(u_{r}\right)_{0}^{k},\left(u_{r}\right)_{1}^{k}, \ldots,\left(u_{r}\right)_{n}^{k}\right)^{T}, \\
U_{r r}^{k} & =\left(\left(u_{r r}\right)_{0}^{k},\left(u_{r r}\right)_{1}^{k}, \ldots,\left(u_{r r}\right)_{n}^{k}\right)^{T} .
\end{aligned}
$$

Then

$$
U_{r}^{k}=\frac{1}{h} D_{1} U^{k}, \quad U_{r r}^{k}=\frac{1}{h^{2}} D_{2} U^{k}
$$

From the initial conditions (2) and Dirichlet boundary conditions (3), we can compute the numerical solution of (1) step by step using the scheme and formulas (16).

\section{Stability Analysis}

Sharma and Singh provided a method to study the ability of the nonlinear partial equation in [20], which we used to study the stability of our scheme. According to (18) and $D_{1}, D_{2}$, the scheme (16) can be rewritten as

$$
\begin{aligned}
u_{i}^{k+1}= & \frac{\tau^{2}}{h^{2}}\left(-\frac{1}{6} u_{i-2}^{k}+\frac{5}{3} u_{i-1}^{k}-3 u_{i}^{k}+\frac{5}{3} u_{i+1}^{k}-\frac{1}{6} u_{i+2}^{k}\right) \\
& +\frac{\tau^{2} \alpha}{h r_{i}}\left(\frac{1}{12} u_{i-2}^{k}-\frac{2}{3} u_{i-1}^{k}+\frac{2}{3} u_{i+1}^{k}-\frac{1}{12} u_{i+2}^{k}\right) \\
& +\tau^{2} g\left(r_{i}, t_{k}\right)+2 u_{i}^{k}-u_{i}^{k-1} .
\end{aligned}
$$

If we set $\tau^{2} / h^{2}=s, \tau^{2} \alpha / h r_{i}=w_{i}$, then the scheme is

$$
\begin{aligned}
u_{i}^{k+1}= & \left(-\frac{1}{6} s+\frac{1}{12} w_{i}\right) u_{i-2}^{k}+\left(\frac{5}{3} s-\frac{2}{3} w_{i}\right) u_{i-1}^{k} \\
& +(-3 s+2) u_{i}^{k}+\left(\frac{5}{3} s+\frac{2}{3} w_{i}\right) u_{i+1}^{k} \\
& +\left(-\frac{1}{6} s-\frac{1}{12} w_{i}\right) u_{i+2}^{k}+\tau^{2} g\left(r_{i}, t_{k}\right)-u_{i}^{k-1} .
\end{aligned}
$$

Therefore we obtained

$$
\begin{aligned}
\left|u_{i}^{k+1}\right| \leq & \left|-\frac{1}{6} s+\frac{1}{12} w_{i}\right|\left|u_{i-2}^{k}\right|+\left|\frac{5}{3} s-\frac{2}{3} w_{i}\right|\left|u_{i-1}^{k}\right| \\
& +|-3 s+2|\left|u_{i}^{k}\right|+\left|\frac{5}{3} s+\frac{2}{3} w_{i}\right|\left|u_{i+1}^{k}\right| \\
& +\left|\frac{1}{6} s+\frac{1}{12} w_{i}\right|\left|u_{i+2}^{k}\right|+\tau^{2}\left|g\left(r_{i}, t_{k}\right)\right|+\left|u_{i}^{k-1}\right| .
\end{aligned}
$$

Taking the norm of (21), we have

$$
\begin{aligned}
\left\|u_{i}^{k+1}\right\|_{L}^{\infty}= & \sup _{i}\left|u_{i}^{k+1}\right| \\
\leq & \sup _{i}\left|-\frac{1}{6} s+\frac{1}{12} w_{i}\right| \sup _{i}\left|u_{i-2}^{k}\right| \\
& +\sup _{i}\left|\frac{5}{3} s-\frac{2}{3} w_{i}\right| \sup _{i}\left|u_{i-1}^{k}\right|+|-3 s+2| \sup _{i}\left|u_{i}^{k}\right| \\
& +\sup _{i}\left|\frac{5}{3} s+\frac{2}{3} w_{i}\right| \sup _{i}\left|u_{i+1}^{k}\right| \\
& +\sup _{i}\left|\frac{1}{6} s+\frac{1}{12} w_{i}\right| \sup _{i}\left|u_{i+2}^{k}\right|+\tau^{2} \sup _{i}\left|g\left(r_{i}, t_{k}\right)\right| \\
& +\sup _{i}\left|u_{i}^{k-1}\right| .
\end{aligned}
$$

Since $\sup _{i} w_{i}=\sup _{i}\left(\tau^{2} \alpha / h r_{i}\right)=\tau^{2} \alpha / h h=s \alpha$ and $\inf _{i} w_{i}=$ $\tau^{2} \alpha / h=w$, thus from (22) we have

$$
\begin{aligned}
\left\|u_{i}^{k+1}\right\|_{L}^{\infty} \leq & \left|-\frac{1}{6} s+\frac{1}{12} w\right|\left\|u_{i}^{k}\right\|_{L}^{\infty}+\left|\frac{5}{3} s-\frac{2}{3} w\right|\left\|u_{i}^{k}\right\|_{L}^{\infty} \\
& +|-3 s+2|\left\|u_{i}^{k}\right\|_{L}^{\infty}+\left|\frac{5}{3} s+\frac{2}{3} s \alpha\right|\left\|u_{i}^{k}\right\|_{L}^{\infty} \\
& +\left|\frac{1}{6} s+\frac{1}{12} s \alpha\right|\left\|u_{i}^{k}\right\|_{L}^{\infty}+\tau^{2} G^{k}+A^{k-1},
\end{aligned}
$$

where $G^{k}=\sup _{i}\left|g\left(r_{i}, t_{k}\right)\right|$ and $A^{k-1}=\sup _{i}\left|u_{i}^{k-1}\right|$. We set $\tau^{2} G^{k} /\left\|u_{i}^{k}\right\|_{L}^{\infty}=d_{L}^{k}, A^{k-1} /\left\|u_{i}^{k}\right\|_{L}^{\infty}=v_{L}^{k-1}$ and $B=\mid-(1 / 6) s+$ $(1 / 12) w|+|(5 / 3) s-(2 / 3) w|+|-3 s+2|+|(5 / 3) s+(2 / 3) s \alpha \mid+$ $|(1 / 6) s+(1 / 12) s \alpha|$; then from (23) we have

$$
\left\|u_{i}^{k+1}\right\|_{L}^{\infty} \leq\left(B+d_{L}^{k}+v_{l}^{k-1}\right)\left\|u_{i}^{k}\right\|_{L}^{\infty} .
$$

It implies that the method is stable if $B+d_{L}^{k}+v_{l}^{k-1} \leq C_{0}$.

\section{Numerical Experiments}

In this section, some numerical solutions of the one-dimensional linear singular hyperbolic equation in the form (1) with the initial conditions (2) and boundary conditions (3) with the scheme (16) are presented.

The versatility and the accuracy of the proposed method is measured using the $L_{2}$ and $L_{\infty}$ error norms for the test problems. The error norms are defined as

$$
L_{2}=\sqrt{h \sum_{j=0}^{n}\left|\left(u_{j}^{e}-u_{j}^{a}\right)\right|^{2}}, \quad L_{\infty}=\max _{0 \leq j \leq n}\left|u_{j}^{e}-u_{j}^{a}\right|,
$$




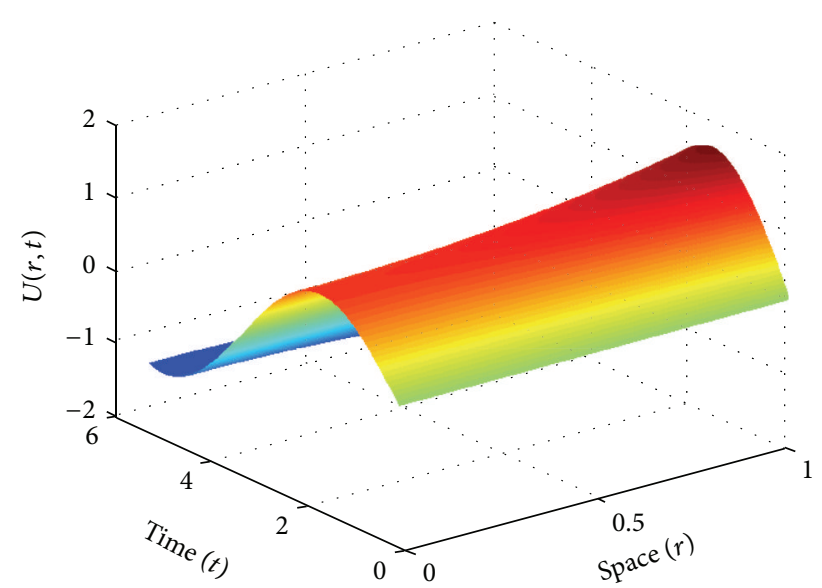

FIgURE 2: Exact solution of Example 1 for $0 \leq r \leq 1$ and $0 \leq t \leq 5$.

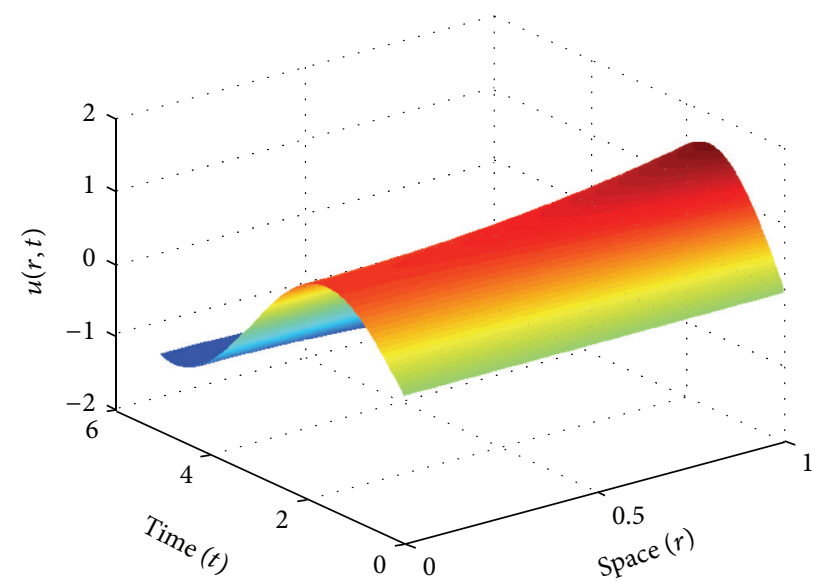

FIgURE 3: Numerical solution of Example 1 using $h=0.005$ and $\tau=$ 0.0005 for $0 \leq r \leq 1$ and $0 \leq t \leq 5$.

where $u_{j}^{e}$ and $u_{j}^{a}$ are the exact and approximate solution of $u$ in $r_{j}$ and arbitrary value of $t$, respectively.

Example 1. In this example, we consider (1) with $g(r, t)=$ $-2 \cosh (r) \sin (t)-(\alpha / r) \sinh (r) \sin (t)$, and $0 \leq r \leq 1, t \geq 0$, $\alpha=1$. The initial condition are given by

$$
\begin{gathered}
u(r, 0)=0, \\
u_{t}(r, 0)=\cosh (r),
\end{gathered}
$$

and the boundary conditions

$$
\begin{gathered}
u(0, t)=\sin (t), \\
u(1, t)=\cosh (1) \sin (t), \quad t \geq 0 .
\end{gathered}
$$

The exact solution of this example is $u(r, t)=\cosh (r) \sin (t)$. The root-mean-square error $L_{2}$ and maximum error $L_{\infty}$ are presented in Table 1 . The space-time graph of the exact and numerical solution up to $t=5$ are shown in Figures 2 and 3 . Absolute error between the numerical and analytical solution is also depicted at all mesh points in Figure 4.

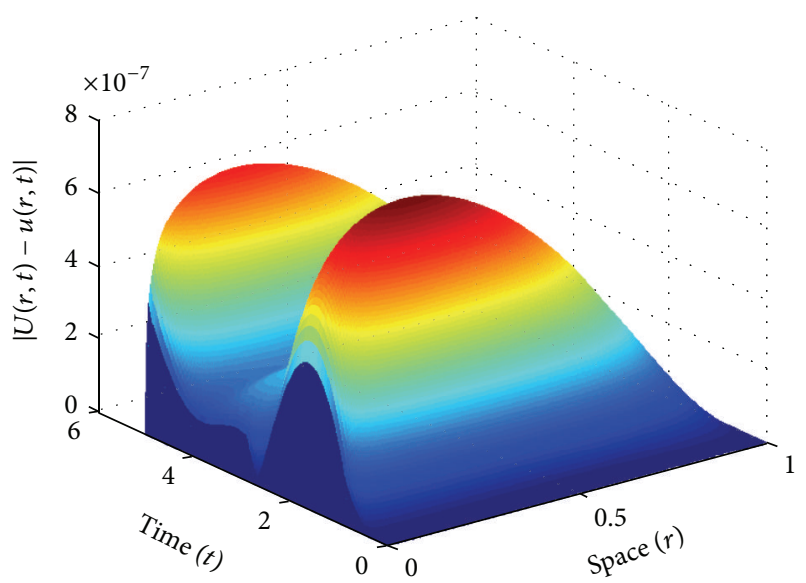

FIgURE 4: Absolute error of Example 1 using $h=0.005$ and $\tau=$ 0.0005 .

TABLE 1: Error norms and invariants $(h=0.005, \tau=0.0005)$.

\begin{tabular}{lcc}
\hline Time & $L_{2}$ error & $L_{\infty}$ error \\
\hline 0.5 & $2.6709 e-08$ & $3.7758 e-08$ \\
1 & $2.5490 e-07$ & $3.2418 e-07$ \\
1.5 & $5.4557 e-07$ & $7.2273 e-07$ \\
2 & $5.2798 e-07$ & $6.8914 e-07$ \\
2.5 & $2.0113 e-07$ & $2.6778 e-07$ \\
3 & $1.3361 e-07$ & $1.9905 e-07$ \\
3.5 & $1.8086 e-07$ & $2.2871 e-07$ \\
4 & $1.5529 e-07$ & $1.9822 e-07$ \\
4.5 & $2.7865 e-07$ & $3.4738 e-07$ \\
5 & $4.9971 e-07$ & $6.5922 e-07$ \\
\hline
\end{tabular}

Example 2. We consider the initial and boundary conditions for (1) as follows:

$$
\begin{gathered}
u(r, 0)=\tanh (r), \quad u_{t}(r, 0)=-\tanh (r), \quad 0 \leq r \leq 1, \\
u(0, t)=0, \quad u(1, t)=e^{-t} \tanh (1), \quad t \geq 0 .
\end{gathered}
$$

In Figures 5 and 6 exact and numerical solutions corresponding to $0 \leq r \leq 1$ and $0 \leq t \leq 5$ are depicted. In our computations, we consider that $g(r, t)=-e^{-t}\left(\alpha+2 r \tanh ^{3}(r)-\right.$ $\left.\alpha \tanh ^{2}(r)-3 r \tanh (r)\right) / r$ and $\alpha=2$. The exact solution of this example is $u(r, t)=e^{-t} \tanh (r)$. The maximum absolute error and the $L_{2}$ norm error, at some time levels, are presented in Table 2. Absolute error between the numerical and analytical solution is also depicted at all mesh points in Figure 7.

Example 3. As a third test problem, we consider (1) with $u(r, t)=\cos (\pi t / 2)(\cosh (r)+\sinh (1-r))$ and $\alpha=1$. The initial condition is given by

$$
\begin{gathered}
u(r, 0)=\cosh (r)+\sinh (1-r), \\
u_{t}(r, 0)=0,
\end{gathered}
$$




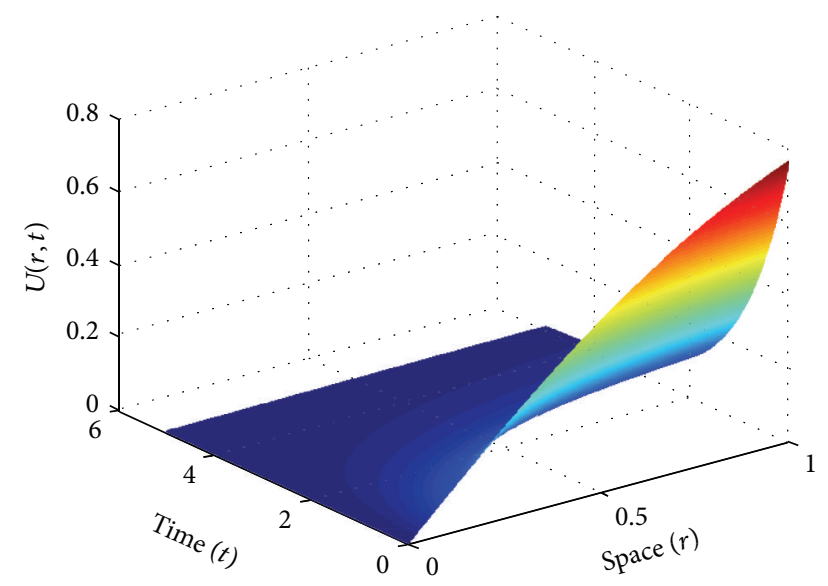

FIgURE 5: Exact solution of Example 2 for $0 \leq r \leq 1$ and $0 \leq t \leq 5$.

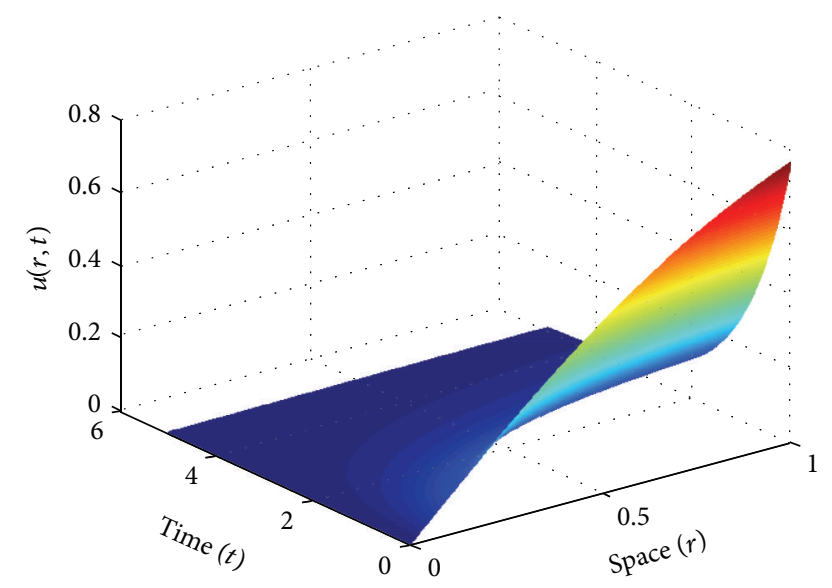

FIgURE 6: Numerical solution of Example 2 using $h=0.005$ and $\tau=0.0005$ for $0 \leq r \leq 1$ and $0 \leq t \leq 5$.

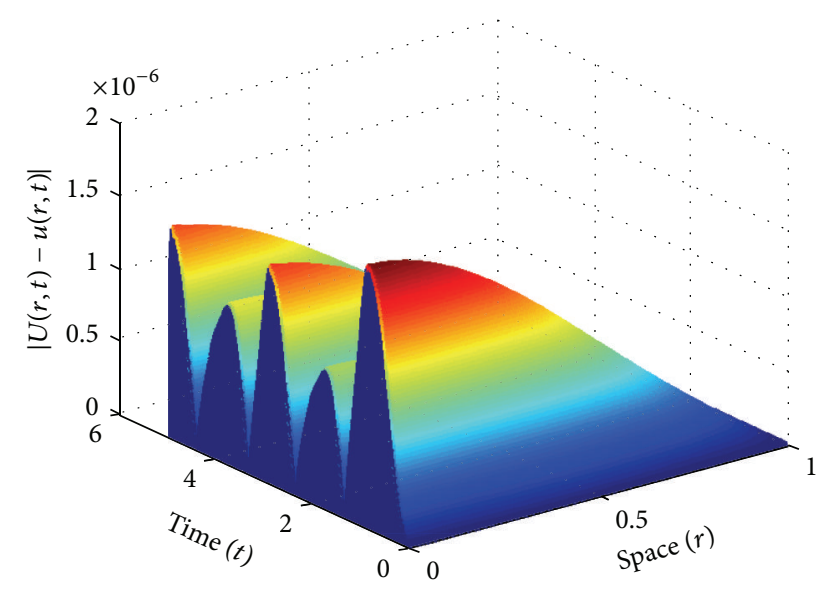

FIgURE 7: Absolute error of Example 2 using $h=0.005$ and $\tau=$ 0.0005 for $0 \leq r \leq 1$ and $0 \leq t \leq 5$.

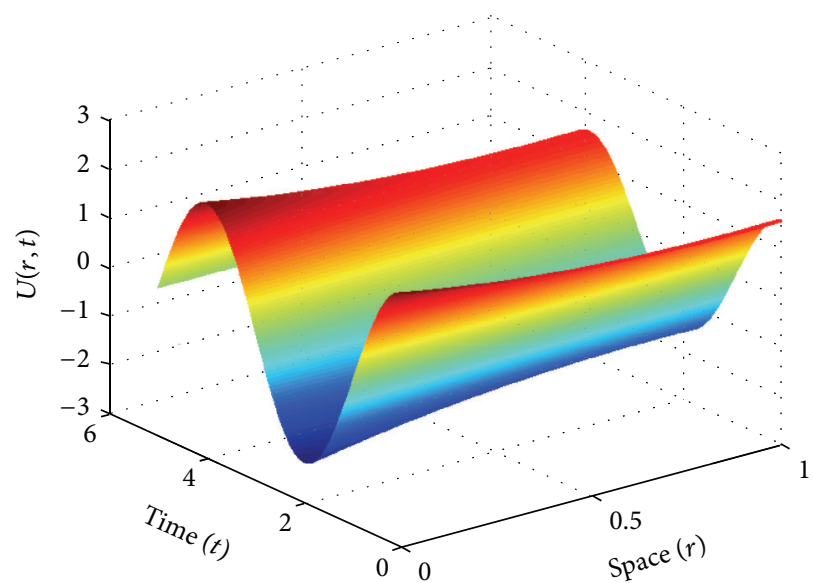

FIgURE 8: Exact solution of Example 3 for $0 \leq r \leq 1$ and $0 \leq t \leq 5$.

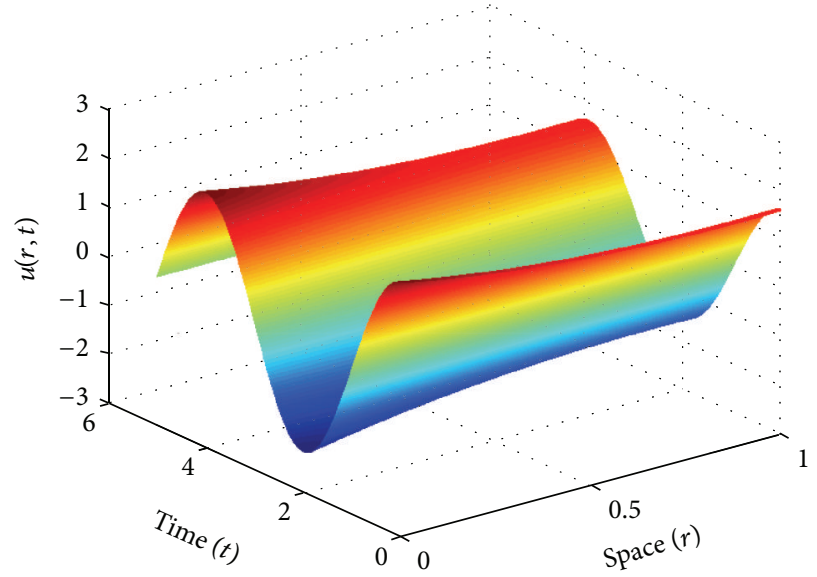

FIGURE 9: Numerical solution of Example 3 using $h=0.005$ and $\tau=0.0005$ for $0 \leq r \leq 1$ and $0 \leq t \leq 5$.

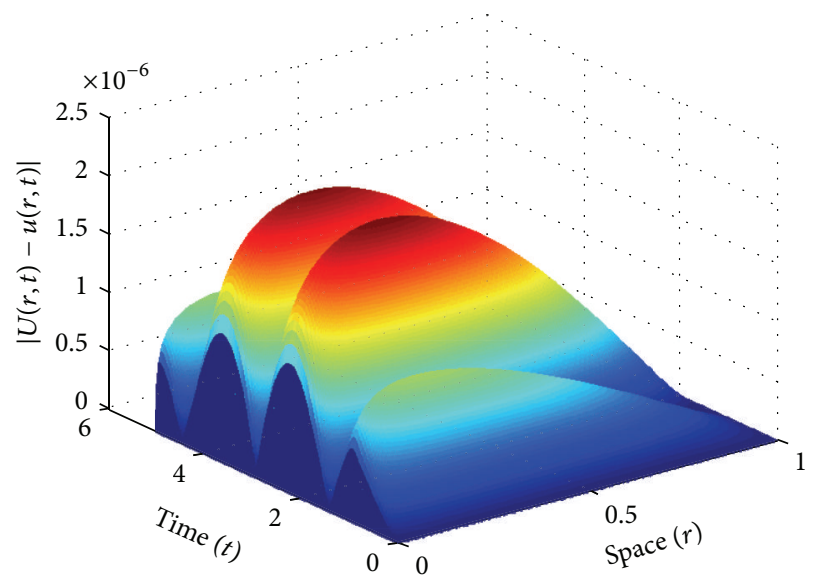

FIgURE 10: Absolute error of Example 3 using $h=0.005$ and $\tau=$ 0.0005 for $0 \leq r \leq 1$ and $0 \leq t \leq 5$. 
TABLE 2: Error norms and invariants $(h=0.005, \tau=0.0005)$.

\begin{tabular}{lcc}
\hline Time & $L_{2}$ error & $L_{\infty}$ error \\
\hline 0.5 & $6.1731 e-07$ & $7.9634 e-07$ \\
1 & $1.1177 e-06$ & $1.7681 e-06$ \\
1.5 & $1.5496 e-07$ & $2.9814 e-07$ \\
2 & $6.1889 e-07$ & $8.4069 e-07$ \\
2.5 & $2.4346 e-07$ & $2.9344 e-07$ \\
3 & $8.9203 e-07$ & $1.4587 e-06$ \\
3.5 & $2.8372 e-07$ & $4.8567 e-07$ \\
4 & $7.0265 e-07$ & $9.5449 e-07$ \\
4.5 & $1.9333 e-07$ & $2.3307 e-07$ \\
5 & $8.6156 e-07$ & $1.4158 e-06$ \\
\hline
\end{tabular}

TABLE 3: Error norms and invariants $(h=0.005, \tau=0.0005)$.

\begin{tabular}{lcc}
\hline Time & $L_{2}$ error & $L_{\infty}$ error \\
\hline 0.5 & $3.6465 e-07$ & $4.4996 e-07$ \\
1 & $7.7447 e-07$ & $1.0921 e-06$ \\
1.5 & $2.2967 e-08$ & $4.3014 e-08$ \\
2 & $1.2812 e-06$ & $1.7105 e-06$ \\
2.5 & $1.4234 e-06$ & $1.9277 e-06$ \\
3 & $2.6966 e-08$ & $3.9557 e-08$ \\
3.5 & $1.4339 e-06$ & $1.9391 e-06$ \\
4 & $1.2373 e-06$ & $1.6524 e-06$ \\
4.5 & $2.8677 e-08$ & $5.6646 e-08$ \\
5 & $7.4448 e-07$ & $1.0497 e-06$ \\
\hline
\end{tabular}

and the boundary conditions

$$
\begin{gathered}
u(0, t)=\cos \left(\frac{\pi t}{2}\right)(1+\sinh (1)), \\
u(1, t)=\cos \left(\frac{\pi t}{2}\right) \cosh (1), \quad t \geq 0 .
\end{gathered}
$$

The space-time graph of the exact and estimated solution up to $t=5$ is presented in Figures 8 and 9. Absolute error between the numerical and analytical solution is also depicted at all mesh points in Figure 10. The root-mean-square error and maximum error are presented in Table 3.

\section{Conclusions}

In this paper, a numerical scheme for the one-dimensional linear singular hyperbolic equation is proposed using cubic B-spline quasi-interpolation. The numerical solutions are compared with the exact solution by finding $L_{2}$ and $L_{\infty}$ errors. From the test examples, we can say that the BSQI scheme is feasible and the error is acceptable. The implementation of the present method is a very easy, acceptable, and valid scheme.

\section{Conflict of Interests}

The authors of the paper do not have a direct financial relation that might lead to a conflict of interests for any of the authors.

\section{References}

[1] W. G. Bickley, "Piecewise cubic interpolation and two-point boundary problems," Computer Journal, vol. 11, no. 2, pp. 206208, 1968.

[2] G. F. Raggett and P. D. Wilson, "A fully implicit finite difference approximation to the one-dimensional wave equation using a cubic spline technique," IMA Journal of Applied Mathematics, vol. 14, no. 1, pp. 75-78, 1974.

[3] T. C. Chawla, G. Leaf, W. L. Chen, and M. A. Grolmes, "The application of the collocation method using hermite cubic spline to nonlinear transient one-dimensional heat conduction problem," Journal of Heat Transfer, vol. 97, no. 4, pp. 562-569, 1975.

[4] S. G. Rubin and P. K. Khosla, "Higher-order numerical solution using cubic splines," AIAA Journal, vol. 14, no. 7, pp. 851-858, 1976.

[5] M. K. Jain and T. Aziz, "Cubic spline solution of two-point boundary value problems with significant first derivatives," Computer Methods in Applied Mechanics and Engineering, vol. 39, no. 1, pp. 83-91, 1983.

[6] H. M. El-Hawary and S. M. Mahmoud, "Spline collocation methods for solving delay-differential equations," Applied Mathematics and Computation, vol. 146, no. 2-3, pp. 359-372, 2003.

[7] R. K. Mohanty, "An unconditionally stable difference scheme for the one-space-dimensional linear hyperbolic equation," Applied Mathematics Letters, vol. 17, no. 1, pp. 101-105, 2004.

[8] A. Mohebbi and M. Dehghan, "High order compact solution of the one-space-dimensional linear hyperbolic equation," Numerical Methods for Partial Differential Equations, vol. 24, no. 5, pp. $1222-1235,2008$.

[9] C.-G. Zhu and R.-H. Wang, "Numerical solution of Burgers' equation by cubic B-spline quasi-interpolation," Applied Mathematics and Computation, vol. 208, no. 1, pp. 260-272, 2009.

[10] L. Ma, Z. Mo, and X. Xu, "Quasi-interpolation operators based on a cubic spline and applications in SAMR simulations," Applied Mathematics and Computation, vol. 217, no. 8, pp. 38533868, 2010.

[11] M. Dosti and A. Nazemi, "Solving one-dimensional hyperbolic telegraph equation using cubic B-spline quasi-interpolation," International Journal of Mathematical \& Computer Sciences, vol. 7, no. 2, p. 57, 2011.

[12] C.-C. Wang, J.-H. Huang, and D.-J. Yang, "Cubic spline difference method for heat conduction," International Communications in Heat and Mass Transfer, vol. 39, no. 2, pp. 224-230, 2012.

[13] M. K. Kadalbajoo, L. P. Tripathi, and A. Kumar, "A cubic Bspline collocation method for a numerical solution of the generalized Black-Scholes equation," Mathematical and Computer Modelling, vol. 55, no. 3-4, pp. 1483-1505, 2012.

[14] S. A. Khuri and A. Sayfy, "A spline collocation approach for a generalized parabolic problem subject to non-classical conditions," Applied Mathematics and Computation, vol. 218, no. 18, pp. 9187-9196, 2012.

[15] R. K. Mohanty, R. Kumar, and V. Dahiya, "Cubic spline iterative method for Poisson's equation in cylindrical polar coordinates," International Scholarly Research Network ISRN Mathematical Physics, vol. 2012, Article ID 234516, 11 pages, 2012.

[16] R. C. Mittal and R. K. Jain, "Numerical solutions of nonlinear Burgers' equation with modified cubic B-splines collocation method," Applied Mathematics and Computation, vol. 218, no. 15, pp. 7839-7855, 2012. 
[17] R. K. Mohanty, R. Kumar, and V. Dahiya, "Cubic spline method for 1D wave equation in polar coordinates," International Scholarly Research Network ISRN Computational Mathematics, vol. 2012, Article ID 302923, 6 pages, 2012.

[18] C. De Boor, A Practical Guide to Splines, Springer, New York, NY, USA, 1978.

[19] P. Sablonnière, "Univariate spline quasi-interpolants and applications to numerical analysis," Rendiconti del Seminario Matematico, vol. 63, no. 3, pp. 211-222, 2005.

[20] K. K. Sharma and P. Singh, "Hyperbolic partial differentialdifference equation in the mathematical modeling of neuronal firing and its numerical solution," Applied Mathematics and Computation, vol. 201, no. 1-2, pp. 229-238, 2008. 


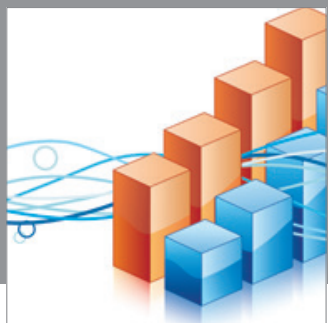

Advances in

Operations Research

mansans

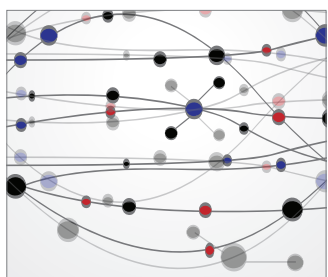

The Scientific World Journal
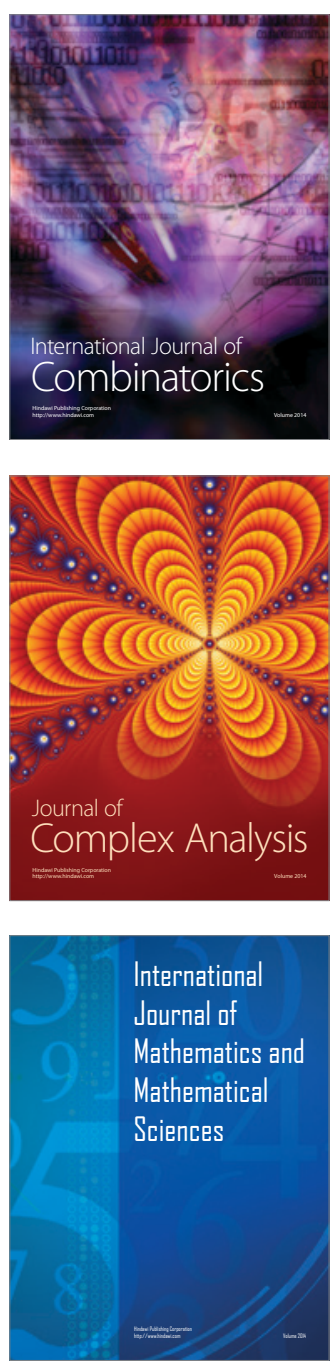
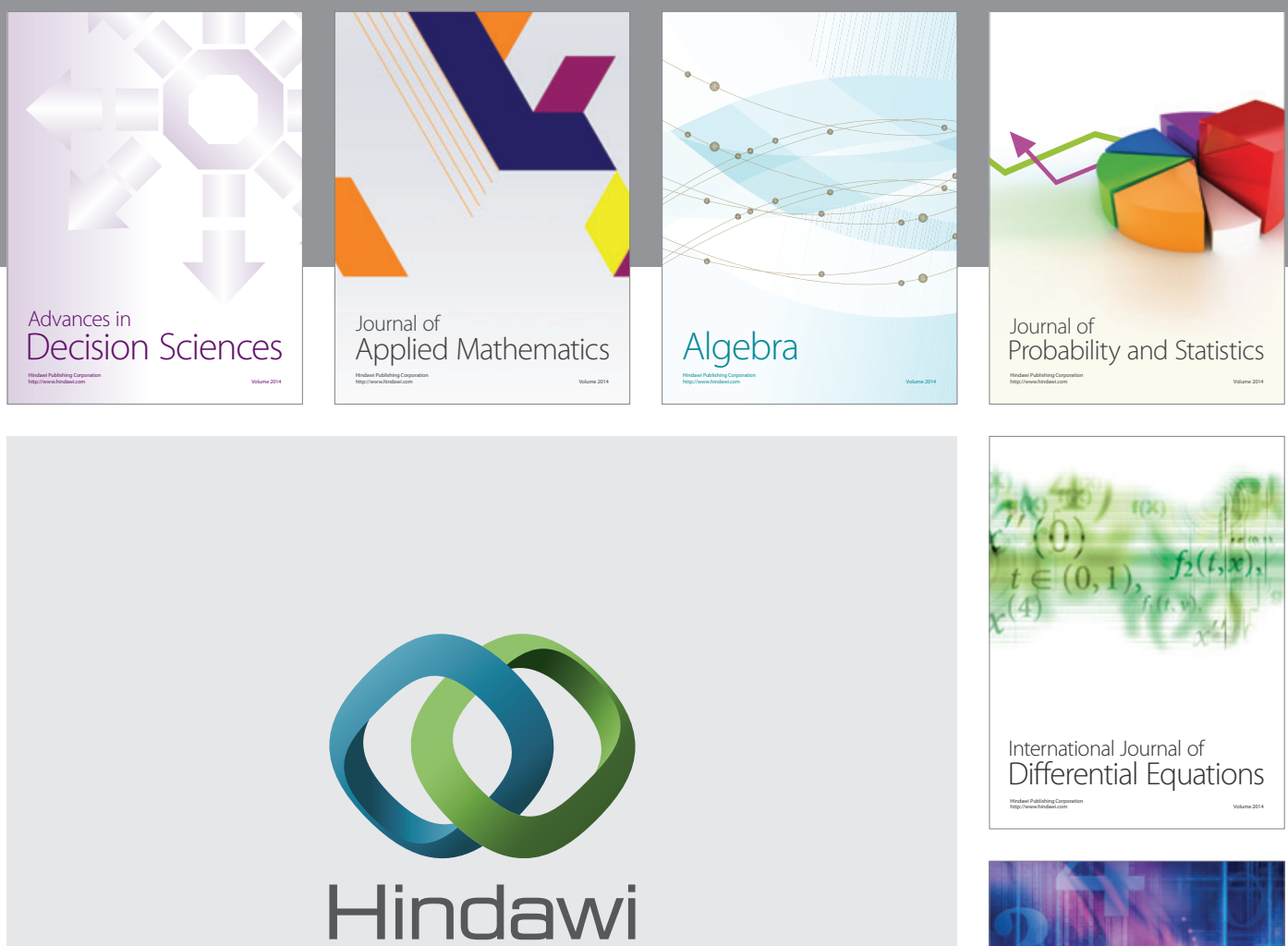

Submit your manuscripts at http://www.hindawi.com
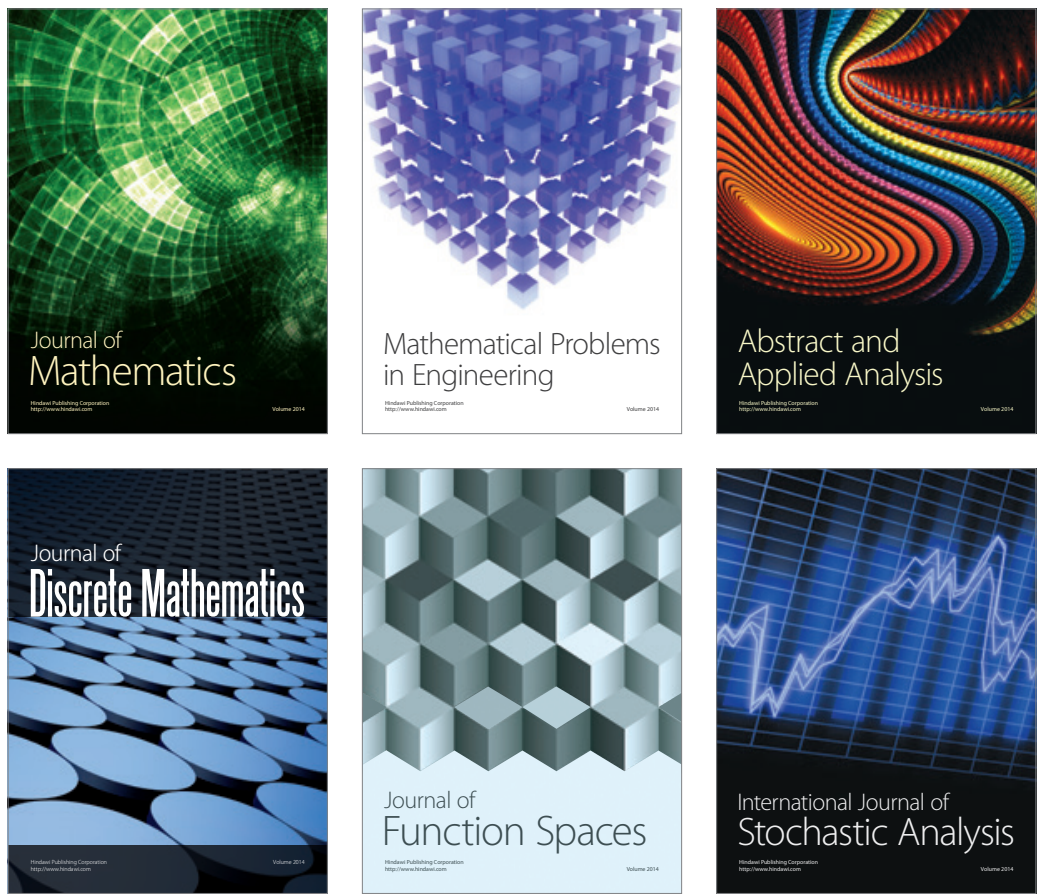

Journal of

Function Spaces

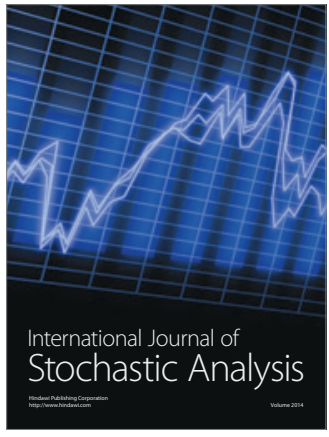

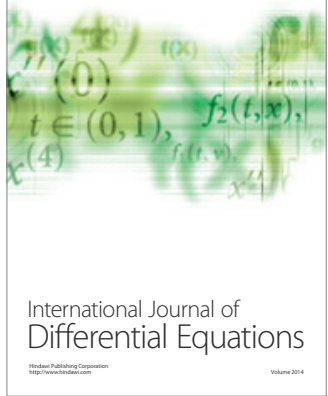
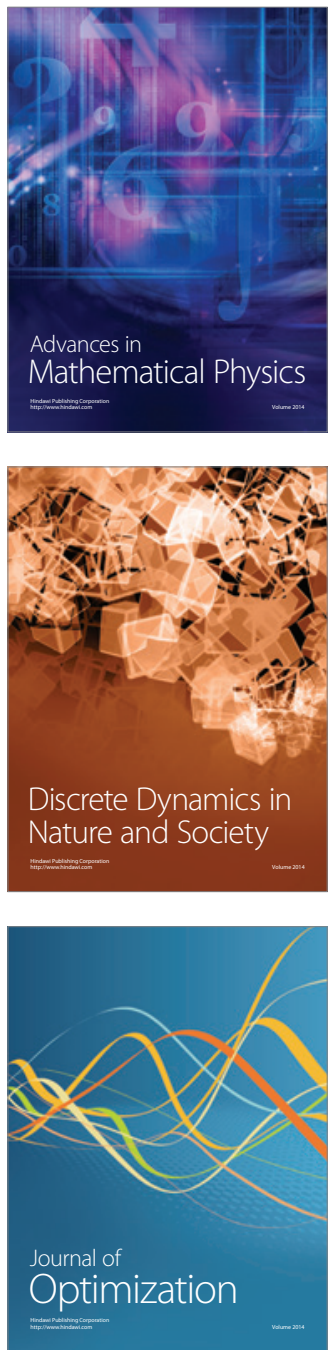